Nawfal A. Zakaria

BDS, M.Sc. (Assist Prof.)

Neam N. Al-Yousifany

BDS, MSc (Lect)

Raghad A. Al-Askry

BDS, M.Sc. (Assist Lect)

\section{Microleakage of Repaired Amalgam}

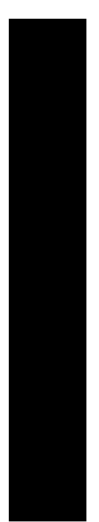

\author{
Department of Conservative Dentistry \\ College of Dentistry, University of Mosul
}

\begin{abstract}
Aims: This invitro study designed to investigate the effect of bonding agent on amalgam repaired with either spherical or admixed alloy. Material and Methods: Cl I cavity preparations were made on the occlusal surface of hundred and twelve extracted human premolars. The teeth were randomly divided into two groups, 56 cavities filled with spherical amalgam and the other 56 cavities filled with admixed amalgam, after aging of restorations, buccal half of the previously placed restorations were removed and each group was divided into two subgroup according to the lining material used. The final number were eight groups of 14 teeth in each. All specimens were thermocycled for 500 times with 15 second dwell time. The samples were stained with $2 \%$ methylene blue, sectioned and evaluated for dye penetration. The data were analyzed by Fisher Freeman Halton test.Results: There was a significant differences in microleakage of bonded and non-bonded specimens, the use of bonding agent significantly increase the microleakage of repaired amalgam. Repairing amalgam with similar alloy type significantly reduce the microleakage.Conclusions: Within the limitation of this study, repairing amalgam with bonding agent is acceptable but significantly less microleakage can observed when amalgam repaired without bonding agents. malgam alloy can repaired with different types of alloy, but better marginal seal accomplished when the same types of alloy used for repair
\end{abstract}

Zakaria NA, Al-Yousifany NN, Al-Askry RA. Microleakage of repaired amalgam. Al-Rafidain Dent J. 2008; 8(1):44-49.

Received: 28/1/2007

Sent to Referees: $17 / 2 / 2007$

Accepted for Publication: 24/4/2007

\section{INTRODUCTION}

Studies on durability of repaired amalgam restoration need to evaluate the amount of microleakage of repaired specimens. Microleakage is defined as the passage of bacteria, fluids, molecules or ions between cavity wall and the restorative material applied to it ${ }^{(1)}$.

Amalgam restorations sometimes fracture due to the faults made during cavity preparations and restorations procedures or where recurrent caries has rendered a portion of them defective ${ }^{(2,3)}$.

Dentists are frequently facing with a clinical decision to replace or repair an amalgam restoration. These situations involve amalgam or cuspal fracture, inadequate marginal integrity and inadequate interproximal contact. The complete replacement of large restorations is time consuming, technically difficult and may be potentially damaging to the pulp ${ }^{(4,5)}$.

There are many factors play a role in the quality of the amalgam repair these factors are time of repair, type of amalgam alloy used in repair, the effect of roughening the fracture surface and the use of adhesive resin. Each of these factors has been evaluated by researchers with little agreement studies. The important factors in the quality of the amalgam repair are the strength of the joined amalgam surfaces and the microleakage at the interface. Microleakage has been recognized as a major clinical problem with direct filled dental restorations $^{(3,5-7)}$.

Because microleakage can cause an extensive damage, much effort has recently been directed toward developed ways to reduce the microleakage including the application of dentinal bonding agents ${ }^{(4)}$.

The purpose of this invitro study was to observe the effects of certain variables, including bonding materials and types of alloy spherical or admixed) on the microleakage between original amalgam and repair interface. 


\section{MATERIALS AND METHODS}

A total of one hundred twelve extracted human maxillary premolar teeth free of cracks (examined by fiber optic light), free of caries and restorations on the visual inspection were selected for the experiment. All teeth were cleaned and polished using scalars and pumice, then stored in distilled water at room temperature prior to use.

The root apex of each tooth was sealed with a self-cured composite restorative material, two coats of nail varnish were applied to the entire external surface of the root, each tooth was mounted in cold curing acrylic resin to the level of cementoenamel junction.

A diamond bur No. 556 in a high speed hand piece with air/water coolant was used to prepare a conventional CL I cavities of $2 \mathrm{~mm}$ width and $2 \mathrm{~mm}$ depth extending to include the mesial and distal pits without undermining the marginal ridges .

The specimens were randomly divided into two groups of fifty six teeth according to the type of amalgam alloy used. Each tooth was washed with water and dried with a compressed air, restored with corresponding amalgam.

Fifty six cavities were filled with a precapsulated spherical alloy ( Vivacap,
Vivadent, Ets., Germany) and another group of (56) cavities were filled with a precapsulated admixed alloy ( Septalloy NG 70, Specialites Septodont, France).

The precapsulated amalgams were triturated with a mechanical amalgamator (Silamat, Vivadent, Schaan/ Liechtenstein, Germany) according to the manufacture instructions. Amalgam immediately condensed into the corresponding cavities by using a mechanical condensation technique with ultra sonic condenser ( Piezon master 400, EMS SA, CH- 1260 Nyon, Switzerland).

Amalgam was placed incrementally, each increment was condensed against the cavity walls for 20 second with a circular flat ultrasonic condenser tip at the medium power until the cavity was over filled, carved to cavosurface margins of the preparation, then finished and burnished. The specimens were stored in distilled water at $37^{\circ} \mathrm{C}$ in an incubator for 30 days. After aging of the restorations, each group was randomly divided into two subgroups of twenty eight teeth according to the amalgam used in repairing procedure , each sub group was divided into two groups of fourteen teeth according to the lining materials used The number of final groups were eight groups as shown in table (1).

Table (1): Diagrammatic illustration of the experimental groups design for the study.

\begin{tabular}{cccc}
\hline Group No. & Type of exist amalgam & Bonding use & Type of fresh amalgam \\
\hline $\mathbf{1}$ & Spherical & No & Spherical \\
$\mathbf{2}$ & Spherical & No & Admixed \\
$\mathbf{3}$ & Spherical & Alloy bond & Spherical \\
$\mathbf{4}$ & Spherical & Alloy bond & Admixed \\
$\mathbf{5}$ & Admixed & No & Admixed \\
$\mathbf{6}$ & Admixed & No & Spherical \\
$\mathbf{7}$ & Admixed & Alloy bond & Admixed \\
$\mathbf{8}$ & Admixed & Alloy bond & Spherical \\
\hline
\end{tabular}


For all specimens half of the previously placed amalgam restorations were removed with a No. 556 diamond bur in a high-speed hand piece with water coolant, the depth of these new preparations also measured $2 \mathrm{~mm}$ in depth ( new diamond bur were replaced after each 6 samples) all preparations were thoroughly washed with water and air dried, each group was treated before insertion of the new amalgam.

For group with lining material used $37 \%$ phosphoric acid for 20 seconds were applied to the prepared cavity and the exist amalgam in each tooth , washed thoroughly with water and air dried gently to keep moist surfaces, alloybond primer applied to the both tooth and previously placed amalgam surfaces, dry with air gently for 2 seconds to evaporate solvent and leave surfaces glossy, light cured for 10 seconds. One drop of alloy bond base mixed with one drop of alloybond catalyst, the mixture was placed in a thin layer on all walls of the preparations, as well as to the previously placed amalgam surface using brush. Freshly triturated amalgam condensed into the non-bonded and bonded cavities immediately using ultra sonic condenser, the restorations were carved and finished to the cavosurface margins of the preparation.

All specimens were thermocycled for 500 times at temperature ranging from $5^{\circ} \mathrm{C} \pm 2^{\circ} \mathrm{C}$ to $55^{\circ} \mathrm{C} \pm 2^{\circ} \mathrm{C}$ each cycle lasted 45 second with a dwell time of 15 seconds in each bath, and 15 seconds intervals between baths, for all specimens the entire tooth surfaces were painted with two coats of nail varnish, except for an area of 1 $\mathrm{mm}$ away from the cavity margins and restoration, to prevent dye penetration anywhere other than the restoration margins. Each layer of varnish was allowed to dry before the next layer was applied.

The samples were immersed in $2 \%$ solution of methylene blue at $37^{\circ} \mathrm{C}$ for (48) hours. After the removal of the teeth from the dye they were washed with copious amount of water to remove any surface traces of dye, dried and were sectioned through the middle of restoration by using a diamond sectioning disks .

The dye penetration at the oldnew amalgam interface was evaluated blind and independently by two evaluators under stereomicroscope (Hamilton by AITAY International Italy) at a magnification level of x10 and scored according to the criteria described by Going etal ${ }^{(8)}$. (Figure.1)

Degree 0 - no penetration of dye.

Degree 1 - penetration of dye up to half of the wall depth.

Degree 2 - penetration of dye to the floor of the cavity but not including it.

Degree 3 - penetration of dye completely surrounding the filling including the cavity floor.

Degree 4 - diffusion of dye into the dentin under the floor or base of the cavity.

Degree 5 - penetration of dye through the dentin to the pulp chamber.

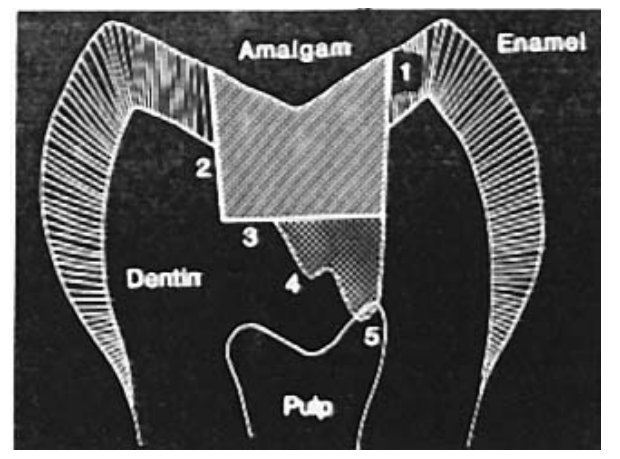

Figure (1): Diagram illustrated the degree of dye penetration

Data were subjected to the Fisher Freeman Halton statistical test at confidence level of $95 \%(\mathrm{p}<0.05)$

\section{RESULTS}

Table (2) shows the distribution of leakage scores between previously placed amalgam and fresh amalgam of the eight test groups.

In general, all specimens show a lower percentage of dye penetration, the microleakage score of all groups were either zero (no dye penetration) or score one (that is mean the dye not reach more than half of the cavity depth).(figure 2).

Fisher Freeman Halton test shows that there was a significant differences in the microleakage between the bonded and non-bonded repaired amalgam, at p value of 0.009. Table (3) Similar statistical analyses were under taken in comparing the two types of alloy used for repair, there was a significant decrease in microleakage at $p$ value of 0.002 when the same type of alloy used for repair. Table (4) 


\section{DISCUSSION}

Numerous investigations have used a variety of research tools to evaluate the extent of microleakage at marginal integrity of restorations, stereomicroscopic examination of dye penetration most com- monly used methods. ${ }^{(9)}$

The results of the present study indicated that the microleakage between the new and existing amalgam surfaces was of score zero (no microleakage) or score one (not more than half of the cavity depth).

Table (2): The frequency of microleakage scores for the eight tested groups.

\begin{tabular}{|c|c|c|c|c|c|c|c|c|c|}
\hline \multirow[b]{2}{*}{ Group No. } & \multirow[b]{2}{*}{ Experimental groups } & \multicolumn{6}{|c|}{ Site of leakage } & \multirow[t]{2}{*}{ Total no. of teeth } & \multirow[t]{2}{*}{ p-value } \\
\hline & & $\mathbf{0}$ & & 2 & 3 & 4 & 5 & & \\
\hline 1 & $S+S$ & 2 & 12 & 0 & 0 & 0 & 0 & 14 & \multirow{8}{*}{$<0.001$} \\
\hline 2 & $\mathrm{~S}+\mathrm{A}$ & 6 & 8 & 0 & 0 & 0 & 0 & 14 & \\
\hline 3 & $S+B+S$ & 8 & 6 & 0 & 0 & 0 & 0 & 14 & \\
\hline 4 & $\mathrm{~S}+\mathrm{B}+\mathrm{A}$ & 2 & 8 & 4 & 0 & 0 & 0 & 14 & \\
\hline 5 & $A+A$ & 8 & 6 & 0 & 0 & 0 & 0 & 14 & \\
\hline 6 & $A+S$ & 4 & 10 & 0 & 0 & 0 & 0 & 14 & \\
\hline 7 & $A+B+A$ & 2 & 12 & 0 & 0 & 0 & 0 & 14 & \\
\hline 8 & $A+B+S$ & 6 & 8 & 0 & 0 & 0 & 0 & 14 & \\
\hline
\end{tabular}

Using Fisher-Freeman-Halton test

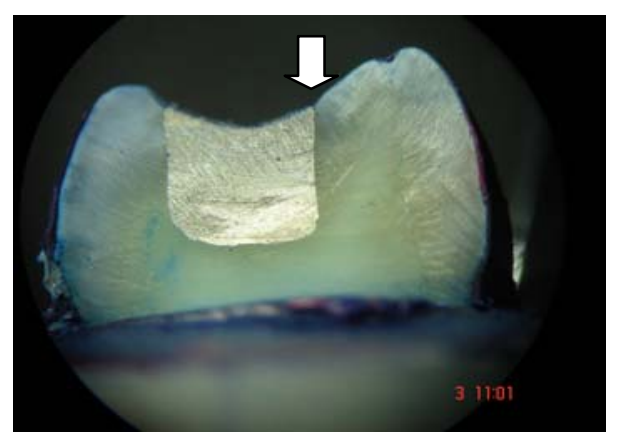

Figure (2): sectioned tooth showing the degree of dye penetration .

Table (3) Statistical analysis comparing the frequency of bonded and nonbonded amalgam.

\begin{tabular}{|c|c|c|c|c|c|}
\hline \multirow{2}{*}{ Score } & \multicolumn{2}{|c|}{ Repair with bonded amalgam } & \multicolumn{2}{|c|}{ Repair without bonded } & \multirow{2}{*}{ p-value } \\
\hline & No. & $\%$ & No. & $\%$ & \\
\hline $\mathbf{0}$ & 18 & 32.1 & 20 & 35.7 & \multirow{6}{*}{0.009} \\
\hline 1 & 34 & 60.7 & 36 & 64.3 & \\
\hline 2 & 4 & 7.2 & 0 & 0.0 & \\
\hline 3 & 0 & 0.0 & 0 & 0.0 & \\
\hline 4 & 0 & 0.0 & 0 & 0.0 & \\
\hline 5 & 0 & 0.0 & 0 & 0.0 & \\
\hline
\end{tabular}

Using Fisher-Freeman-Halton test

Table (4) Statistical analysis for types of amalgam used in repair. 


\begin{tabular}{|c|c|c|c|c|c|}
\hline \multirow[t]{2}{*}{ Score } & \multicolumn{2}{|c|}{ Repair with the same type } & \multicolumn{2}{|c|}{ Repair with different type } & \multirow{2}{*}{ p-value } \\
\hline & No. & $\%$ & No. & $\%$ & \\
\hline $\mathbf{0}$ & 24 & 42.9 & 14 & 25.0 & \multirow{6}{*}{0.002} \\
\hline 1 & 32 & 57.1 & 38 & 67.9 & \\
\hline 2 & 0 & 0.0 & 4 & 7.1 & \\
\hline 3 & 0 & 0.0 & 0 & 0.0 & \\
\hline 4 & 0 & 0.0 & 0 & 0.0 & \\
\hline 5 & 0 & 0.0 & 0 & 0.0 & \\
\hline
\end{tabular}

The exact mechanism is not understood, but the cause may be due to the linear coefficient of thermal expansion of amalgam is about two to three times that of surrounding tooth structure, thus thermal changes not affect the sealing capacity between the fresh and existing amalgam.

The bonding between the two amalgam probably cause from free mercury in fresh amalgam combine with the unconsumed gamma particles in older alloy, this cause a union between them, by the formation of new intermetalic compound. ${ }^{(10,11)}$

Ozer et al. ${ }^{(3)}$ concluded that microleakage of repaired restoration was lower in the amalgam groups than microleakage in the resin composite and composer groups.

A clinical study on survival of repaired amalgam, concluded that repair of local defects in amalgam restoration was an effective alternative to restoration replacement especially up to five years. ${ }^{(12)}$

Shirani et al. study the effect of adhesive system on the microleakage at the amalgam repaired, the results indicated a decrease in the microleakage only when composite resin used for repair. ${ }^{(13)}$

The stress rising from the polymerization shrinkage may results in bond failure and show a greater effects on the marginal adaptation . (14)

The intermediate materials and substrate have a significant effect on sealing ability of amalgam restoration. ${ }^{(15)}$

Our results agree with the results of Fruite et al. who found that strongest repair of amalgam material can accomplished without the use of any bonding agents. ${ }^{(16)}$

The proper technique of condensing amalgam to the surface of an old amalgam important to establish a bond between the new and old alloy. ${ }^{(17)}$
The results indicated better marginal seal when the same type of alloy used for repair. Dispers alloy had the least microleakage and tytin amalgam had the highest microleakage. ${ }^{(10)}$

Spherical alloy has coarse-grained texture that formed surface channels through which microleakage can more rapidly occur. Also may have more intervolumetric space which may contribute to more microleakage. ${ }^{(18,19)}$

In clinical practice it is not always possible for clinician to determine the specific type of dental amalgam.

The leakage around amalgam does not depend solely on the shape of particles, condensation force was reported as the most important factor influencing adaptation of amalgam alloy to cavity walls and as well as to the old amalgam. ${ }^{(11)}$

Heavy and constant condensational force in the present study was due to the use of ultrasonic condenser which produced a better adaptation for all types of amalgam alloy.

Amalgam alloy show reduce in the microleakage overtime, this reduction has been explained by corrosion products filling the interface gap in order to seal the restoration. ${ }^{(20)}$

Amalgam restoration is still the best material used for amalgam repair even without bonding material.

From clinical point, repair cause less damage than complete replacement of amalgam and it is therefore preferred.

\section{CONCLUSION}

The bonding agent significantly increase the microleakage so it is preferable not to use bonding in amalgam repair. Even with bonding the microleak-age is accepted . 
The use of the same amalgam alloy is preferable because it gives better marginal adaptation.

Although this invitro study designed to simulate the clinical environment, but further testing need, especially (clinical investigations) to assess the real longevityof repair restorations.

\section{REFERENCES}

1. Kimuro Y. Yonago K, Yokoyama, Matsuoka, Saki K and Matsumoto K. Apical leakage of obturated canals prepared by Er. YAG laser. Am Assoc Endod 2001; 27: 567-579.

2. Walker AC and Reese SB. Bond strength of amalgam to amalgam in a high-copper amalgam. Oper Dent. 1983; 8: 99-102.

3. Ozer F, Unlu N, Ozturk B and Sengun A. Amalgam repair: evaluation of bond strength and microleakage. Oper Dent. 2002; 27: 299-303.

4. Hadavi F, Hey JH, Ambrose ER and Elbadrawy HE. Repair of high-copper amalgam with and without an adhesive system: Invitro assessment of microleakage and shear bond strength. General Dent. 1993; 41: 49-58.

5. Jessup JP, Vandewalle KS, Hermesch CB and Buikema DJ. Effect of surface treatment on amalgam repair. Oper Dent. 1998; 23: 15-20.

6. El-Housseiny AA and Farsi N. Sealing ability of a single adhesive in primary teeth: an in vivo study. Int $J$ Paediat Dent. 2002; 12: 265-270.

7. Giannini M, Paulillo LA and Ambrosano GM. Effect of surface roughness on amalgam repair using adhesive systems. Braz Dent J 2002; 13: 179-183.

8. Going RE, Massler $M$ and Dute HL. Marginal penetration of dental restoration as studied by crystal violet dye. J Am Dent Assoc. 1960; 61: 283-300.

9. Delnero MO and Dela Macorra JC. Sealing and dentin bond strength of adhe- sive system. Oper Dent. 1999; 24: 194 202.

10. Chang J, Chan J, Cheda H and Iglesias A. Microleakage of a 4-methacryloxyethyl trimellitate anhydride bonding agent with amalgam. J. Prosthet Dent. 1996; 75: 495498.

11. Korale ME and Meiers JG. Microleakage of dentin bonding systems used with spherical and admixed amalgams. Am J Dent. 1996; 249-252.

12. Smales J, Howthorne WS. Long term survival of repaired amalgam, recemted crowns and gold casting. Oper Dent. 2004; 29: 249-253.

13. Shirani F, Malekipour MR, Zakouchaki PM and Zia P. Effect of different adhesive system on microleakage at the amalgam. J Isfahan Dent School. 2005; (1)304: 23-29.

14. Smales RJ, Wetherell JD. Review of bonded amalgam restorations and assessment in a general practice over five years. Oper Dent. 2000; 25: 374-381.

15. Dasilva AF, Piva E, Demarco FF, Corrersobrinho L, Osinga PW. Microleakage in conventional and bonded amalgam restorations influence of cavity volume. Oper Dent. 2006; 31(3): 377-383.

16. Fruits TJ, Duncanson MGJR and Coury TL. Interfacial bond strength of amalgam bonded to amalgam and resin composite bonded to amalgam. Quintessence Int. 1998; 29(5): 327-334.

17. Shen C. Speigel J. and Major LA, Repair strength of dental amalgam. Oper Dent. 2006; 31(1): 122-126.

18. Fayyad MA and Ball PC. Cavity sealing ability of lath-cut, blend and spherical amalgam alloy: a laboratory study. Oper Dent. 1984; 9: 86-93.

19. Mahler DB. The high-copper dental amalgam alloy. J Dent Res. 1997; 76: 537-541.

20. Osborne JW. Creep as a mechanism for sealing amalgam. Oper Dent. 2006; 31(2): 161-164. 